\title{
Caractérisation et étude de l'impact sur l'environnement de sédiments dragués dans le nord de la France
}

\author{
Rachid ZENTAR, Nor-Edine ABRIAK, Ngoc Thanh TRAN
}

École des Mines de Douai, Département Génie Civil \& Environnemental, 941 rue Charles Bourseul, 59500 Douai Cedex, France.

zentar@ensm-douai.fr

\section{Résumé :}

Ce travail s'intègre dans le cadre plus général d'une méthodologie proposée pour optimiser la gestion des sédiments marins et fluviaux dragués dans le nord de la France. La première étape consiste à déterminer leurs caractéristiques en relation avec le domaine de valorisation envisagé, puis à déterminer leurs propriétés physiques, minéralogiques et chimiques. Leurs impacts sur l'environnement sont étudiés par le biais d'essais de lixiviation. Les résultats obtenus permettent de proposer leur utilisation, sous réserve, dans le domaine des travaux routiers.

Soumis le 28 janvier 2008, accepté le 27 novembre 2008, en ligne le 9 février 2009.

La seule version examinée est celle écrite en français. La ou les autres versions n'étant pas examinées par le comité de rédaction de la revue, sont donc publiées sous l'entière responsabilité du ou des auteurs.

A TRANSLATED VERSION IN ENGLISH IS AVAILABLE ONLINE

Pour citer cet article :

ZENTAR R., ABRIAK N.-E., TRAN N.T. (2009). Caractérisation et étude de l'impact sur l'environnement de sédiments dragués dans le nord de la France. Revue Paralia, $\mathrm{n}^{\circ} 2$, pp 4.1-4.12. 


\section{Introduction}

Le dragage consiste à déplacer ou à enlever un matériau de son environnement aquatique. L'opération de dragage peut être nécessaire afin de maintenir ou d'améliorer les conditions de navigation par (1) l'approfondissement ou l'élargissement des chenaux marins, (2) le désenvasement des ports, (3) la création de nouvelles voies de navigation et (4) pour l'enlèvement des sédiments pollués de l'environnement aquatique.

Avec l'augmentation de la demande de matériaux granulaires dans divers domaines comme dans les travaux de génie civil, l'industrie, l'agriculture ou la réhabilitation de sites (CENTRE SAINT-LAURENT, 1993 ; ULBRICHT, 2002 ; LIFE, 2002), l'utilisation des sédiments dragués peut améliorer la gestion des ressources naturelles de granulats en constituant une nouvelle source de matériaux.

En France, le génie civil consomme annuellement plus de 400 millions de tonnes de matériaux granulaires dont plus de la moitié dans le domaine des travaux routiers (UNPG, 2005; MICHEL, 1997). Pour assurer une valorisation convenable des sédiments dragués, il est fondamental de connaître leurs caractéristiques géotechniques. De plus et en raison de la présence dans ces sédiments de matière organique et de polluants (et de sel en milieu marin), l'évaluation de leurs impacts sur l'environnement est également d'une importance majeure.

Cet article concerne deux sédiments du nord de la France, l'un marin et l'autre fluvial. Leurs caractéristiques physiques, minéralogiques et chimiques vont être établies en fonction d'une valorisation en travaux routiers, tandis que leur impact potentiel sur l'environnement sera évalué au travers d'essais de lixiviation.

\section{Méthodologie}

La méthodologie développée pour assurer la valorisation des sédiments dragués dans le domaine des travaux routiers se décompose en trois étapes (ZENTAR et al., 2005) :

- Une étape de caractérisation des sédiments bruts qui inclut l'identification des caractéristiques mécaniques, physico-chimiques et minéralogiques des sédiments bruts. Elle comprend également l'étude de leur impact potentiel sur l'environnement.

- Une étape de formulation du matériau routier qui a pour rôle l'amélioration des performances mécaniques des sédiments bruts pour atteindre les performances souhaitées. Elle inclut une phase de caractérisation des matériaux d'ajouts utilisés et l'étude de la formulation du matériau routier.

- Une étape de validation qui comprend la validation des caractéristiques mécaniques et l'étude des incidences sur l'environnement du matériau formulé.

Plus précisement l'accent sera mis ici sur l'identification des caractéristiques physicochimiques et minéralogiques des sédiments bruts et sur l'étude de l'impact sur l'environnement des matériaux formulés dans le contexte d'une valorisation dans le domaine routier. La technique de formulation et les caractéristiques mécaniques des 
matériaux formulés, décrites par Dubois (DUBOIS, 2006), ne seront que très brièvement abordées dans cet article.

\section{Présentation des sites de prélèvement}

Les sédiments étudiés dans ce travail sont issus du port de Dunkerque pour le sédiment marin et du canal de la Scarpe Supérieure pour le sédiment fluvial (Fig. 1).

Dunkerque, troisième port Français en terme d'échange de marchandises, assure un trafic annuel de plus de 57.11 millions de tonnes de marchandises. Concernant l'activité sédimentaire, près de 4 millions de $\mathrm{m}^{3}$ de boues y sont dragués tous les ans (DUBOIS, 2006). La proportion principale de ces sédiments est considérée comme non polluée, selon la législation française (METL \& MATE, 2000). Dans ce cas, leur immersion est toujours possible. Mais le sédiment marin étudié provient de l'est du port. Or ce secteur est considéré comme pollué selon l'arrêté du 14/06/2000, fondé sur une étude de zonage (MAC FARLANE, 2004).

La Scarpe Supérieure s'étend entre Arras et Corbehem dans la région du Pas-de-Calais. Le prélèvement a été réalisé au milieu du canal, à proximité de la ville de Brebières située à environ 50 kilomètres de la ville de Lille (France).
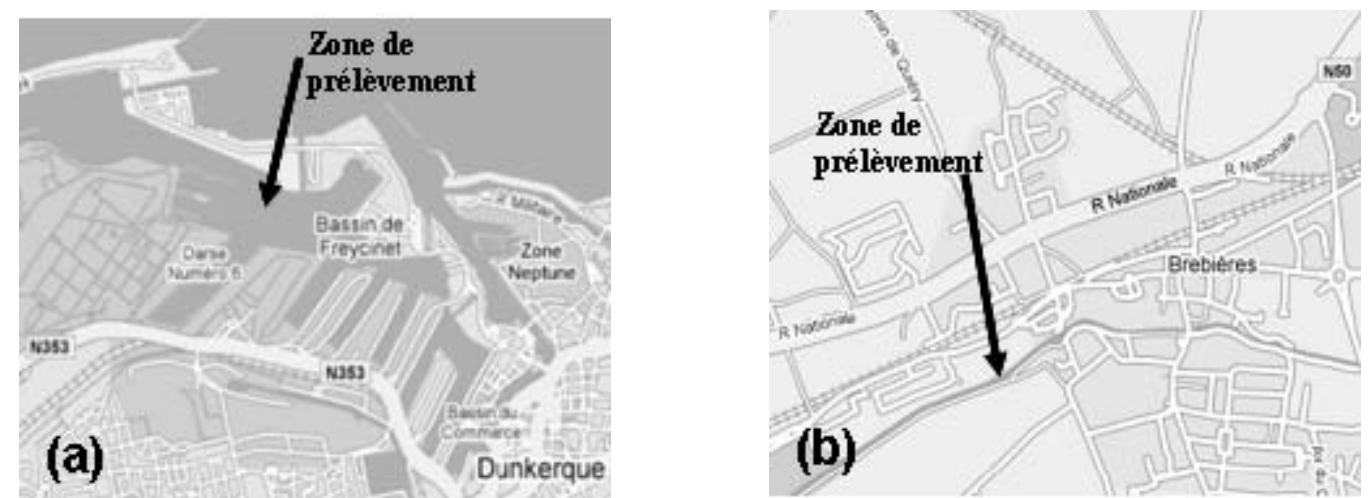

Figure 1. Zones de prélèvement du sédiment marin (a) et du sédiment fluvial (b).

\section{Résultats d'essais et discussion}

\subsection{Détermination des caractéristiques physiques}

Dans le domaine des travaux routiers, la détermination de la granulométrie, la quantité et l'activité de la fraction d'argile, la teneur en matière organique, et les limites de consistance des matériaux constituent le point de départ de toute étude. La connaissance de ces caractéristiques permet d'appliquer la classification française des matériaux selon les spécifications du guide des terrassements routiers (GTR, 1992).

Dans cette étude, la distribution granulométrique des sédiments a été déterminée par la technique de diffraction laser. Des résultats obtenus, il s'avère que les deux types de sédiments sont comparables. Ils se composent principalement de particules fines. La 
proportion d'argile granulométrique (particules inférieures à $2 \mu \mathrm{m}$ ) est légèrement plus importante pour le sédiment fluvial (12\%) que pour le sédiment marin (9\%), (tableau 1). Pour caractériser l'activité de la fraction argileuse, la méthode d'adsorption du bleu de méthylène est utilisée (NF P 94-068, 1993). Pour chaque type de sédiment, la valeur moyenne de trois résultats d'essai est reportée dans le Tableau 1. De ces résultats, il apparaît que l'activité de la fraction argileuse du sédiment fluvial est sensiblement plus importante que celle de la fraction argileuse du sédiment marin. Ce résultat est partiellement dû au taux plus élevé d'argiles dans le sédiment fluvial, comparé à celui du sédiment marin, mais également au type de minéraux argileux comme il sera montré par les analyses de diffraction de rayons $\mathrm{X}$.

La teneur en matières organiques (MO), pour les deux types de sédiment, a été déterminée en utilisant deux méthodes : Par la méthode de perte au feu à $450{ }^{\circ} \mathrm{C}$ (XP P 94-047, 1998) et par la méthode d'oxydation sulfochromique (NF ISO 14235, 1998). Dans la deuxième méthode, la teneur en MO est déduite de la teneur en carbone organique en multipliant cette dernière quantité par 1.72 (MUSTIN, 1987). D'après les valeurs présentées dans le tableau 1, les résultats d'essais semblent être comparables pour le sédiment marin où une teneur en MO d'environ $8 \%$ est estimée avec les deux méthodes mais dans le sédiment fluvial, la différence relative observée avoisine les $100 \%$. La surestimation de la teneur en MO par la méthode de la perte au feu qui additionne tous les départs de substances volatiles, a pu être induite ici par le cumul de la destruction de la MO et du départ de l'eau liée aux argiles minéralogiques : on sait que cette perte d'eau est plus prononcée pour les minéraux argileux à surface spécifique élevée (HOLTZAPFFEL, 1985).

Les limites d'Atterberg des deux types de sédiment sont déterminées selon l'essai NF P 94-051 (1993). La limite de liquidité $\left(\mathrm{w}_{\mathrm{L}}\right)$ est mesurée en utilisant l'appareillage de Casagrande et la limite de plasticité $\left(\mathrm{w}_{\mathrm{P}}\right)$ par la technique des rouleaux. En terme de $\mathrm{w}_{\mathrm{P}}$, les valeurs mesurées sur les deux types de sédiment sont de l'ordre de $30 \%$. Pour la $\mathrm{w}_{\mathrm{L}}$, la valeur mesurée sur le sédiment marin est presque deux fois supérieure à la valeur mesurée sur le sédiment fluvial. Selon une étude complémentaire réalisée sur le sédiment marin, il a été montré que cette valeur élevée de $\mathrm{w}_{\mathrm{L}}$ est principalement due à la présence de MO (DUBOIS, 2006). Sur la base de la méthode de détermination de la MO par oxydation sulfochromique, la teneur en MO du sédiment fluvial est moitié moindre que la teneur mesurée dans le sédiment marin.

En plus des quatre caractéristiques physiques de base décrites et mesurées ci-dessus, la masse volumique des particules solides $\left(\rho_{\mathrm{s}}\right)$ a été déterminée à l'aide d'un pycnomètre à hélium. Pour chaque type de sédiment, les résultats rapportés dans le tableau 1 sont les valeurs moyennes de 240 mesures effectuées sur trois échantillons. En termes de résultats, comme prévu, les valeurs mesurées sont inférieures aux valeurs connues pour les particules minérales constituant les sédiments $\left(\approx 2.70 \mathrm{t} / \mathrm{m}^{3}\right)$. Cette différence est due principalement à la présence de $\mathrm{MO}$ de moindre densité. Pour vérifier ce constat, on a 
mesuré celle des particules solides, après traitement à $450{ }^{\circ} \mathrm{C}$ pendant $12 \mathrm{~h}$. Le résultat obtenu est comparable aux valeurs standard des matériaux inorganiques. Aussi, la différence observée entre les valeurs de $\rho_{\mathrm{s}}$ mesurées pour les échantillons traités à $450{ }^{\circ} \mathrm{C}$ et les sédiments bruts est plus importante pour le sédiment marin que pour le sédiment fluvial. Ce résultat pourrait soutenir l'hypothèse que le sédiment fluvial à une teneur en MO inférieure à celle dans le sédiment marin.

En termes de classification des matériaux, selon le guide des travaux routiers (GTR, 1992), le sédiment marin et le sédiment fluvial étudiés sont considérés comme des matériaux modérément organiques. Ceci est dû à la teneur en matière organique (supérieure à 3\%). Cette classe de matériaux est dénotée comme la classe F. D'après la distribution granulométrique, la valeur du bleu de méthylène et les limites d'Atterberg, le sédiment marin est classé comme un matériau de type A4-F11. Cette classe englobe les sols argileux qui sont modérément organiques. Le sédiment fluvial est classé comme un matériau de type A2-F11; cette classe englobe les sols limoneux qui sont modérément organiques (GTR, 1992).

Dans le domaine des travaux routiers, l'utilisation de ces matériaux en remblais de très faible hauteur est permis dans le meilleur des cas. Pour un usage dans la structure des chaussées, des essais additionnels sont nécessaires pour une meilleure évaluation du comportement mécanique.

Tableau 1. Caractéristiques physiques des sédiments étudiés.

\begin{tabular}{|c|c|c|}
\hline Paramètres & $\begin{array}{c}\text { Sédinent } \\
\text { marin }\end{array}$ & $\begin{array}{c}\text { Sedintent } \\
\text { fluvial }\end{array}$ \\
\hline$\%<2 \mu m(A r g i l e s)$ & $9 \%$ & $12 \%$ \\
\hline $2 u m<\%<6 \mu m($ Limons $)$ & $72 \%$ & $80 \%$ \\
\hline $63 u m<\%($ Sables $)$ & $19 \%$ & $8 \%$ \\
\hline Valew au blen (VBS) & 3.4 & 42 \\
\hline \% Mo par perte au feu & 8.6 & 7.2 \\
\hline$\%$ Mo par oxydation & 7.2 & 3.1 \\
\hline$w_{I}(\%)$ & 89.4 & 49.5 \\
\hline$w F(\%)$ & 35.5 & 28.0 \\
\hline$I F(\%)$ & 539 & 21.5 \\
\hline$\rho_{2}\left(t / m^{3}\right)$ & 253 & 2.56 \\
\hline$\rho_{5}\left(t \mathrm{~m}^{3}\right)$ après tratement $\left(450^{\circ} \mathrm{C}\right)$ & 2.69 & 2.66 \\
\hline
\end{tabular}

4.2 Analyse minéralogique

Une bonne compréhension de la composition minéralogique des matériaux est essentielle pour anticiper les problèmes qui pourraient être induits lors de la valorisation des sédiments dragués et peut aussi expliquer les contradictions observées dans les résultats d'essais courants comme dans l'essai de perte au feu, l'oxydation 
sulfochromique et l'essai au bleu de méthylène. Dans cette étude, les phases cristallines dans les matériaux sont identifiées par l'utilisation de la technique de diffraction de rayons X. L'équipement utilisé est du type Siemens D500.

Les trois phases cristallines principales non argileuses du sédiment marin sont la calcite, le quartz et l'halite. La calcite et le quartz dominent dans le sédiment fluvial.

Pour déterminer la nature des phases argileuses, des analyses spécifiques ont été effectuées sur des particules de tailles inférieures à $2 \mu \mathrm{m}$. Ces analyses consistent à caractériser l'échantillon brut, le matériau traité thermiquement à $450{ }^{\circ} \mathrm{C}$ pendant deux heures et le matériau saturé par des vapeurs d'éthylène-glycol. Le traitement thermique facilite la détection de la kaolinite et le traitement par des vapeurs d'éthylène-glycol celle des smectites.

La répartition des phases argileuses est reportée dans le tableau 2 en fonction du traitement des spectres de diffraction obtenus. De ces résultats, il apparaît que la phase argileuse du sédiment marin se compose de smectites, d'illite et de kaolinite en proportions égales.

Les smectites constituent la phase majoritaire du sédiment fluvial. Ce dernier point peut expliquer (1) la valeur élevée précédemment obtenue à partir de ce matériel dans l'essai au bleu de méthylène, tout comme (2) une partie de la différence observée sur ce même sédiment fluvial entre les résultats de l'essai de perte au feu et l'analyse par oxydation sulfochromique. En effet la présence de smectite joue en augmentant la perte au feu, mais reste sans effet dans la seconde méthode.

Tableau 2. Type et proportion de minéraux dans les sédiments étudiés.

\begin{tabular}{lcccc}
\hline Mineraux & Smectites & Illite & Kaolinite & Chlorite \\
\hline Sédiment marin (\%) & 30 & 32 & 30 & 8 \\
Sédiment fluvial (\%) & 55 & 20 & 15 & 10 \\
\hline
\end{tabular}

\subsection{Aspect environnemental}

Pour évaluer les impacts sur l'environnement de ces sédiments bruts, on a réalisé des essais de lixiviation selon les spécifications définies dans la norme européenne (NF EN12457-2, 2002), en prenant un rapport liquide sur solide de 10.

Les paramètres inorganiques, tels que les métaux et les anions, sont déterminés comme les concentrations dans les lixiviats. Les paramètres organiques, tels que les hydrocarbures aromatiques polycycliques (HAP), les diphényles d'huile minérale et polychlorés ( $\mathrm{PCBs}$ ), le benzène, le toluène, le benzène éthylique et les composés de xylène (BTEX) sont déterminés directement sur le solide. Les seuils prescrits dans la décision européenne (CUE, 2003) sont utilisés pour évaluer l'impact potentiel des matériaux sur l'environnement. Cependant il est important de noter que ces seuils ont été précisement développés pour établir des critères pour l'acceptation de déchets dans 
les centres d'enfouissement. Par contre, il n'existe pas pour le moment de seuils spécifiques à la valorisation des sédiments de dragage dans les travaux routiers.

Les résultats des analyses sur les deux types de sédiments sont récapitulés dans le tableau 3. A partir de ces résultats, il apparaît que les teneurs respectives en chlorures, en molybdène et en hydrocarbures pour le sédiment marin, et en HAP pour le sédiment fluvial, dépassent les limites fixées pour les déchets inertes. Il est important de noter que les essais sur le sédiment marin ont été réalisés sur un matériau à teneur en eau de mer de $200 \%$.

\begin{tabular}{|c|c|c|c|}
\hline \multicolumn{4}{|c|}{ Essaisur lixiviats } \\
\hline Eléments & Unité & $\begin{array}{c}\text { Sédiment } \\
\text { marin }\end{array}$ & $\begin{array}{c}\text { Sédiment } \\
\text { fluvial }\end{array}$ \\
\hline$p H$ & & 8.3 & 8.0 \\
\hline Conductivité & $\mathrm{mS} / \mathrm{cm}$ & 7.7 & 0.2 \\
\hline As & $m g / \mathrm{kg}$ & $<0.5$ & $<0.5$ \\
\hline $\mathrm{Ba}$ & $\mathrm{mg} / \mathrm{kg}$ & $<0.2$ & $<0.2$ \\
\hline $\mathrm{Cd}$ & $m g / k g$ & $<0.04$ & $<0.04$ \\
\hline $\mathrm{Cr}$ & $m g / \mathrm{kg}$ & $<0.5$ & $<0.5$ \\
\hline$C u$ & $m g / k g$ & $<0.5$ & $<0.5$ \\
\hline $\mathrm{Hg}$ & $m g / k g$ & $<0001$ & $<0001$ \\
\hline Mo & $\mathrm{mg} / \mathrm{kg}$ & 4.19 & $<0.5$ \\
\hline $\mathrm{Ni}$ & $m g / k g$ & $<0.4$ & $<0.4$ \\
\hline $\mathrm{Pb}$ & $m g / k g$ & $<0.5$ & $<0.5$ \\
\hline$S b$ & $m g / h g$ & $<0.06$ & $<0.06$ \\
\hline Se & $m g / h g$ & $<0.1$ & $<0.1$ \\
\hline$Z n$ & $m g / h g$ & 0.73 & $<0.5$ \\
\hline Finorures & $m g / k g$ & 7.1 & 2.5 \\
\hline Chlorures & $m g / h g$ & 27537 & 30 \\
\hline Sulfates & $m g / \mathrm{kg}$ & 3718 & 310 \\
\hline Indice Phenol & $m g / h g$ & 0.64 & $<0.10$ \\
\hline COT & $m g / h g$ & 479 & 78 \\
\hline \multicolumn{4}{|c|}{ Essai sur sobide } \\
\hline COT & $m g / h g$ & 42000 & 18000 \\
\hline$B T E X$ & $m g / h g$ & $<5$ & $<5$ \\
\hline$P C B$ & $m g / h g$ & 0.28 & 0055 \\
\hline Hydrocarbures & $m g / k g$ & 820 & 230 \\
\hline$H A P$ & $m g / k g$ & 29.1 & 110 \\
\hline
\end{tabular}

Tableau 3. Résultats de l'analyse des sédiments bruts. 
L'étude menée pour déterminer les caractéristiques mécaniques des sédiments bruts pour une utilisation dans le domaine des travaux routiers, aspect qui n'est pas abordé dans cet article, a indiqué une capacité portante relativement faible, une sensibilité élevée à l'eau et des caractéristiques mécaniques médiocres. Afin d'assurer une valorisation économiquement viable, les performances mécaniques ont été augmentées en modifiant la distribution granulaire des sédiments bruts en ajoutant un sable de carrière (SC) et/ou un sable de dragage (DS) en combinaison avec un liant.

A partir de ces études préliminaires, la quantité de sédiments dans le mélange à été contrainte par le taux de MO acceptable dans le mélange $(<3 \%)$ et par la quantité de particules fines $(<12 \%)$. La teneur en ciment a été fixée à une valeur de $6 \%$ matière sèche, typiquement utilisée dans la profession.

On a ainsi réalisé quatre mélanges à partir de l'étude préliminaire (tableau 4). Deux mélanges sont à base de sédiment marin, avec ou sans sable de carrière (dénommés FM1 et FM2) et deux autres à base de sédiment fluvial (dénommés FF1 et FF2).

Tableau 4. Formulation des matériaux à base du sédiment marin et du sédiment fluvial.

\begin{tabular}{lcccc}
\hline & FM1 & FM2 & FF1 & FF2 \\
\hline \% Sédiment marin & 33 & 33 & $/$ & $/$ \\
\% Sédiment fluvial & $/$ & $/$ & 23 & 23 \\
\% Sable de dragage & 20 & 61 & 24 & 71 \\
\% Sable de carrière & 41 & $/$ & 47 & $/$ \\
\% Ciment & 6 & 6 & 6 & 6 \\
\hline
\end{tabular}

En termes de comportement mécanique, qui n'est pas abordé dans cet article, les essais réalisés sur les mélanges ont permis de valider la méthodologie de formulation.

En ce qui concerne l'étude de l'impact sur l'environnement, les essais de lixiviation sont réalisés sur des échantillons sur éprouvette monolithe (NF X31-211, 2000) et sur des échantillons broyés (NF EN12457-2, 2002). Les monolithes sont préparés de la même manière que les éprouvettes pour essais mécaniques (NF P98-230-2, 1993).

A partir des résultats des analyses (tableau 5), il apparaît que les échantillons monolithes n'indiquent pas de pollution particulière dans les lixiviats, excepté une quantité modérée de chlorures pour les mélanges qui incluent le sédiment marin.

Pour les échantillons broyés, les essais sur les mélanges à base de sédiment fluvial (FF1 et FF2) indiquent que les matériaux sont inertes par comparaison aux seuils établis pour les déchets. Pour les mélanges à base du sédiment marin (FM1 et FM2), les analyses indiquent des quantités de molybdène et de chlorures inférieures à celles mesurées sur le sédiment brut. Toutefois, la teneur en cuivre est sensiblement plus élevée que la teneur mesurée sur le sédiment brut. Ce résultat nécessite des analyses complémentaires et l'analyse des ajouts utilisés dans les mélanges. De façon générale, en fonction des teneurs en chlorures mesurée et du molybdène, les matériaux à base du sédiment marin 
dépassent le seuil des déchets inertes et sont considérés en tant que déchets non dangereux.

Tableau 5. Résultats d'analyse des lixiviats des matériaux formulés à base de sédiments marin et fluvial.

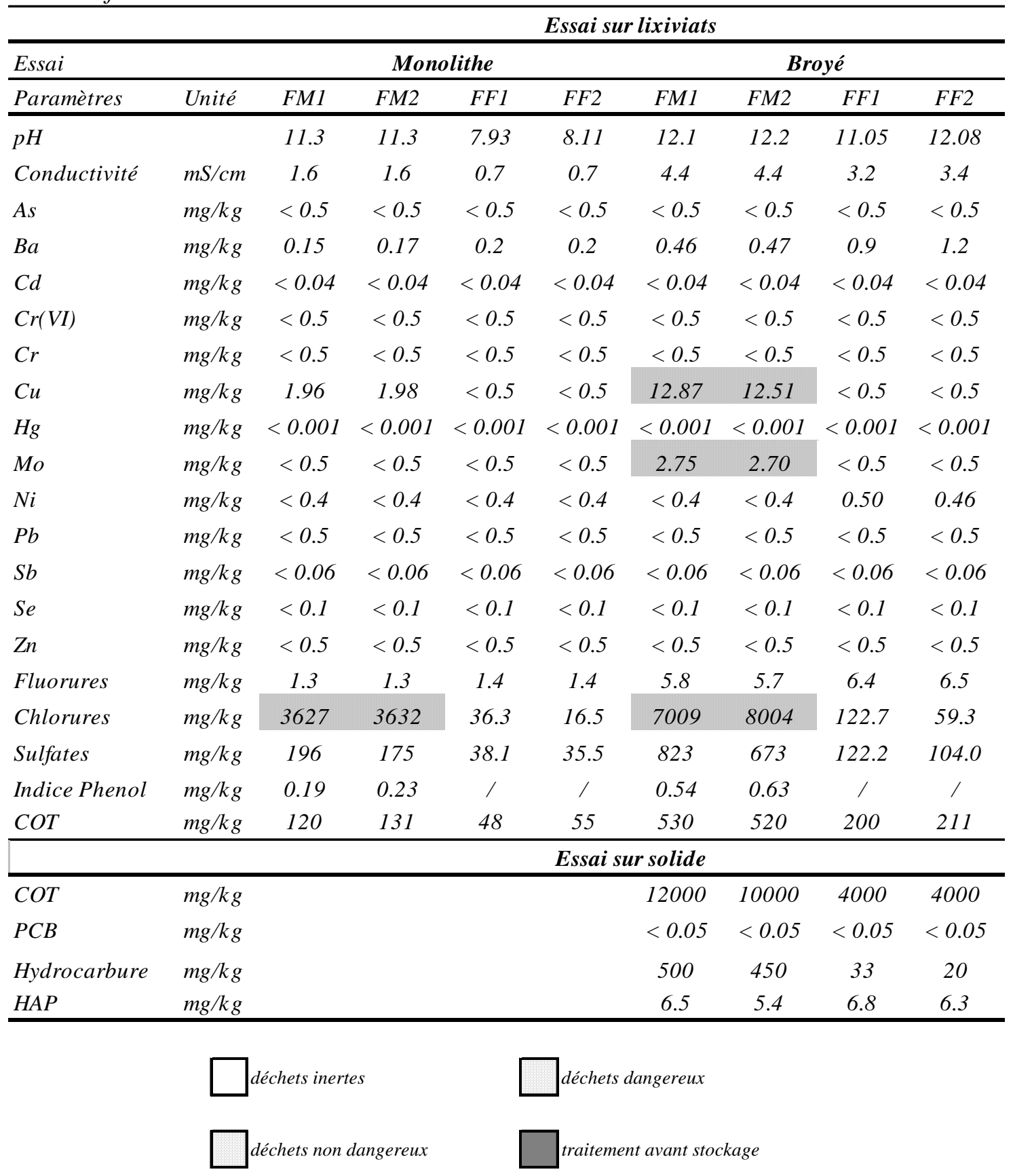

\section{Conclusions}

Les sédiments de dragage, selon la classification européenne (CUE, 2001 ; MATE, 2002), sont considérés comme des déchets. Pour favoriser leur valorisation dans les travaux routiers, il est nécessaire de mieux connaître leurs caractéristiques physiques, 
minéralogiques, chimiques et mécaniques mais aussi il est important d'évaluer leurs impacts sur l'environnement.

Dans cette étude, les caractéristiques physiques principales mesurées montrent que les sédiments étudiés sont des matériaux fins, composés principalement de limons (tamisât à $63 \mu \mathrm{m}$ supérieur à $80 \%$ ), modérément organiques et fortement plastiques. L'impact sur l'environnement, évalué au travers d'essais de lixiviation, montre une teneur élevée de chlorures, des teneurs modérées de molybdène et d'hydrocarbures pour le sédiment marin. Pour le sédiment fluvial, seule la présence des HAP est à signaler.

Pour répondre aux exigences en termes de caractéristiques mécaniques pour la valorisation en technique routière des sédiments dragués, il était nécessaire d'améliorer la distribution granulaire des sédiments en ajoutant du sable et des liants.

Sur les matériaux formulés, les essais de lixiviation réalisés sur les échantillons monolithiques n'ont pas indiqué de pollution particulière, à part une teneur modérée de chlorures pour les matériaux à base de sédiment marin. Pour les essais réalisés sur les échantillons broyés, on observe des teneurs modérées de cuivre, de molybdène et d'hydrocarbures pour les formulations à base de sédiment marin. Les mélanges à base de sédiment fluvial n'ont pas indiqué de problèmes particuliers. Cependant, il est important de noter dans cette étude que les seuils utilisés pour évaluer la qualité des lixiviats sont établis pour l'acceptation de déchets dans les centres d'enfouissement et qu'à présent il n'existe pas de seuils spécifiques pour la valorisation des sédiments de dragage dans des travaux routiers.

A ce stade de l'étude, pour les mélanges qualifiés en termes de comportement mécanique et d'innocuité sur l'environnement, il semble important de valider leurs comportements à l'échelle d'un site.

\section{Remerciements}

Les travaux présentés ci-dessus ont été conduits dans le cadre d'un projet initié pour développer des solutions alternatives à l'immersion pour une meilleure gestion des sédiments dragués. Le projet a été supporté par la région du "Nord-Pas-Calais".

\section{Références bibliographiques}

CENTRE SAINT-LAURENT (1993). Guide pour l'évaluation et le choix des technologies de traitement des sédiments contaminés. Montréal. pp 10-227.

CUE (2001). -Conseil de l'Union Européenne- 2001/573/CE : Décision du Conseil du 23 juillet 2001 modifiant la décision 2000/532/CE de la Commission en ce qui concerne la liste de déchets. Journal Officiel de l'Union Européenne n L 203 du 28/07/2001. pp 0018-0019.

CUE (2003). -Conseil de l'Union Européenne- 2003/33/CE : Décision du conseil du 19 décembre 2002 établissant des critères et des procédures d'admission des déchets dans 
Caractérisation et étude de l'impact sur l'environnement de sédiments dragués dans le nord de la France : 4.11

les décharges, conformément à l'article 16 et à l'annexe II de la directive 1999/31/CE. Journal Officiel de l'Union Européenne n L 011 du 16/01/2003. pp 0027-0049.

DUBOIS V. (2006). Etude du comportement physico-mécanique et caractérisation environnementale des sédiments marins. Thèse de l'Université d'Artois. 285 p.

GTR (1992). Réalisation des remblais et des couches de forme. Guide technique. Fascicule I. Principes généraux.

HOLTZAPFFEL T., (1985). Les minéraux argileux, préparation, analyse diffractométrique et détermination. Publication $\mathrm{n}^{\circ} 12$, Société Géologique du Nord. ISSN 0291-3062

LIFE (2002). Méthodes de gestion et de réutilisation des sédiments pollués. Projet européen réalisé par In Vivo. Agence de l'eau Artois Picardie. Pôle de Compétence des sites et sols pollués. $126 \mathrm{p}$.

MAC FARLANE F. (2004). Méthodologie de gestion des matériaux de dragage maritime - Application au port de Dunkerque. Thèse de l'Université d'Artois. 254 p.

MATE (2002). -Ministre de l'aménagement du territoire et de l'environnement- Décret $n^{\circ}$ 2002-540 du 18/04/2002 relatif à la classification des déchets.. J.O.R.F. $\mathrm{n}^{\circ} 93$ du 20 avril 2002. pp 7074-7088.

METL, MATE (2000). -Ministre de l'équipement, des transports et du logement, Ministre de l'aménagement du territoire et de l'environnement- Arrêté du 14/06/00 relatif aux niveaux de référence à prendre en compte lors d'une analyse de sédiments marins ou estuariens présents en milieu naturel ou portuaire. J.O.R.F. $\mathrm{n}^{\circ} 184 \mathrm{du} 10$ août 2000. pp 12415-12416.

MICHEL F. (1997). Les granulats. Union Nationale des Producteurs de Granulats. France. 1997. $40 \mathrm{p}$.

MUSTIN M. (1987). Le compost. Editions François Dubusc. 954 p.

NF EN 12457-2, Décembre 2002. Caractérisation des déchets. Lixiviation. Essai de conformité pour la lixiviation des déchets fragmentés et des boues. Partie 2 : essai en bâchée unique avec un rapport liquide - solide de 10l/kg et une granularité inférieure à $4 \mathrm{~mm}$ (sans ou avec réduction de la granularité).

NF ISO 14235, Septembre 1998. Qualité du sol. Dosage du carbone organique par oxydation sulfochromique.

NF P 94-068, Novembre 1993. Sols : reconnaissance et essais - mesure de la quantité et de l'activité de la fraction argileuse - détermination de la valeur au bleu de méthylène d'un sol par l'essai à la tâche.

NF P 94-051, Mars 1993. Sols : reconnaissance et essais - détermination des limites d'Atterberg - limite de liquidité à la coupelle - limite de plasticité au rouleau.

NF P 98-230-2, Aout 1993, Essais relatifs aux chaussées - Préparation des matériaux traités aux liants hydrauliques ou non traités - Partie 2 : fabrication des éprouvettes de sables ou de sols fins par compression statique. 
4.12 : Revue Paralia - $n^{\circ} 2$ (2009)

NF X 31-211, Avril 2000. Déchets - Essais de lixiviation d'un déchet solide initialement massif ou généré par un procédé de solidification.

ULBRICHT J.P. (2002). Contaminated sediments: raw materials for bricks. Symposium dragage. Dunkerque. France.

UNPG (2005). -Union Nationale des Producteurs de Granulats- Le marché des granulats en 2004.

XP P94 047. Décembre 1998. Sols : reconnaissance et essais - Détermination de la teneur pondérale en matières organiques d'un matériau. Méthode par calcination.

ZENTAR R.. DUBOIS V., ABRIAK N.E. (2005). Physical and mechanical behaviour of dredged marine sediments. Third international conference on remediation of contaminated sediments. New Orleans. Etats-Unis (LA). 24-27 January 2005. 